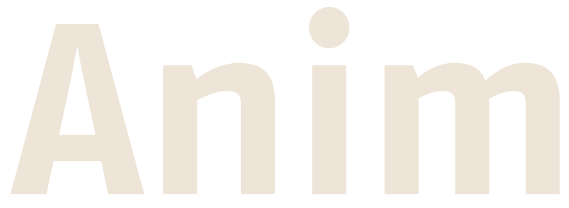

Animals rely upon their acoustic and vibrational senses and abilities to detect the presence of both predators and prey and to communicate with members of the same species. This chapter surveys the physical bases of these abilities and their evolutionary optimization in insects, birds, and other land animals, and in a variety of aquatic animals other than cetaceans, which are treated in Chap. 20. While there are many individual variations, and some animals devote an immense fraction of their time and energy to acoustic communication, there are also many common features in their sound production and in the detection of sounds and vibrations. Excellent treatments of these matters from a biological viewpoint are given in several notable books $[19.1,2]$ and collections of papers [19.3-8], together with other more specialized books to be mentioned in the following sections, but treatments from an acoustical viewpoint [19.9] are rare. The main difference between these two approaches is that biological books tend to concentrate on anatomical and physiological details and on behavioral outcomes,

\subsection{Optimized Communication}

Since animals use their acoustic and vibrational senses both to monitor their environment and to communicate with other animals of the same species, we should expect that natural selection has optimized these sensing and sound production abilities. One particular obvious optimization is to maximize the range over which they can communicate with others of the same species. Simple observation shows that small animals generally use high frequencies for communication while large animals use low frequencies - what determines the best choice? The belief that there is likely to be some sort of universal scaling law involved goes back to the classic work of D'Arcy Thompson [19.10], while a modern overview of physical scaling laws (though not including auditory communication) is given by West and Brown [19.11].

\section{Animal Bioacoustics}

19.1 Optimized Communication 785

19.2 Hearing and Sound Production 787

19.3 Vibrational Communication ................ 788

19.4 Insects.................................... 788

19.5 Land Vertebrates ........................... 790

19.6 Birds .......................................... 795

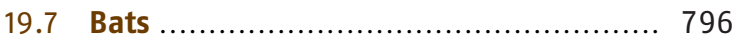

19.8 Aquatic Animals ........................... 797

19.9 Generalities .................................. 799

19.10 Quantitative System Analysis ............. 799

References ...................................... 802

while acoustical books use simplified anatomical models and quantitative analysis to model wholesystem behavior. This latter is the approach to be adopted here.
The simplest assumption is that the frequency is determined simply by the physical properties of the sound-producing mechanism. For a category of animals differing only in size, the vibration frequency of the sound-producing organ depends upon the linear dimensions of the vibrating structure, which are all proportional to the linear size $L$ of the animal, and upon the density $\rho$ and elastic modulus $E$ of the material from which it is made. We can thus write that the song frequency $f=A \rho^{x} E^{y} L^{z}$, where $A, x, y$, and $z$ are constants. Since the dimensions of each side of the equation must agree, we must have $x=-1, y=1$ and $z=-1$, which leads to the conclusion that song frequency should be inversely proportional to the linear size of the animal or, equivalently, that $f \propto M^{-1 / 3}$, where $M$ is the mass of the animal. This simple result agrees quite well with 


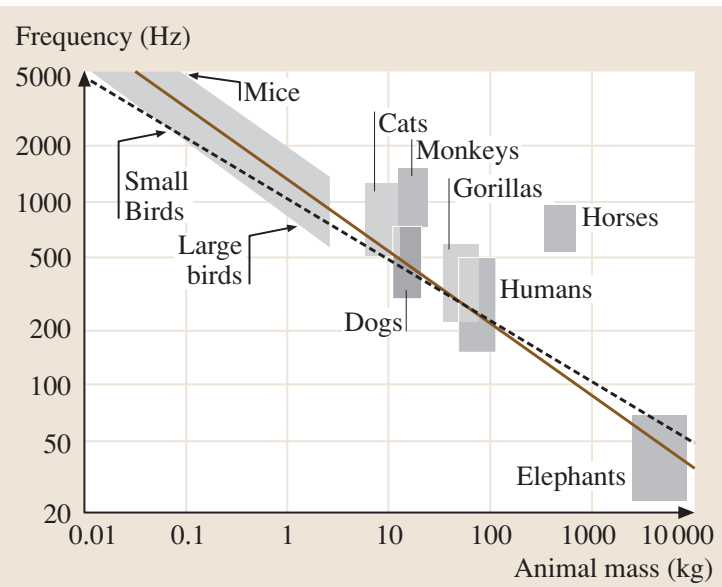

Fig. 19.1 The frequency ranges of the emphasized frequencies of vocalization in a large range of land-dwelling animals, plotted as a function of the mass of the animal. The dashed line shows the regression $f \propto M^{-0.33}$ while the solid line is the regression $f \propto M^{-0.4}$, as discussed in the text [19.12]

observation, as shown by the dashed line in Fig. 19.1, for a wide variety of animals, but a more detailed analysis is desirable.

Over reasonable distances in the air, sound spreads nearly hemispherically, so that its intensity decays like $1 / R^{2}$, where $R$ is the distance from the source. But sound is also attenuated by atmospheric absorption, with the attenuation coefficient varying as $f^{2}$. An optimally evolved animal should have maximized its communication distance under the influence of these two effects. An analysis [19.12] gives the result that $f \propto M^{-0.4}$ which, as shown in Fig. 19.1, fits the observations even better than the simpler result. There are, however, many outliers even among the animals considered, due to different anatomies and habitats. Insects, which must produce their sound in an entirely different way as is discussed later, are not shown on this graph, but there is a similar but not identical relative size relation for them too.

The total sound power produced by an animal is also a function of its size, typically scaling about as $M^{0.53}$ for air-breathing animals of a given category. When the variation of song frequency is included, this leads [19.12] to a conspecific communication distance proportional about to $M^{0.6}$. Again, while this trend agrees with general observations, there are many very notable outliers and great differences between different types of animals. Thus, while mammals comparable in size with humans typically produce sound power in the range $0.1-10 \mathrm{~mW}$, and large birds may produce comparable power, some small insects such as cicadas of mass not much more than $1 \mathrm{~g}$ can also produce almost continuous calls with a power of $1 \mathrm{~mW}$, as will be discussed in Sect. 19.4. At intermediate sizes, however, many animals, particularly reptiles, are almost mute.

Elephants represent an interesting extreme in the animal world because of their very large mass - as much as 10 tonnes. Their calls, which have a fundamental in the range $14-35 \mathrm{~Hz}$, can have an acoustic power as large as $5 \mathrm{~W}$, leading to a detection distance as large as $5 \mathrm{~km}$, or even up to $10 \mathrm{~km}$ after sunset on a clear night [19.13] when very low frequency propagation is aided by atmospheric inversion layers (Chap. 4). Vegetation too, of course, can have a significant effect upon transmission distance. These elephant calls are often misleadingly referred to as "infrasonic" in the biological literature, despite the fact that only the fundamental and perhaps the second harmonic satisfy this criterion, and the calls have many harmonics well within the hearing range of humans. Indeed, even other elephants depend upon these upper harmonics to convey information, and usually cannot recognize the calls of members of the same family group above a distance of about $1.5 \mathrm{~km}$ because of the attenuation of harmonics above about $100 \mathrm{~Hz}$ through atmospheric absorption [19.14].

When it comes to sound detection, rather similar scaling principles operate. As will be discussed briefly in Sect. 19.2, the basic neuro-physiological mechanisms for the conversion of vibrations to neural impulses are remarkably similar in different animal classes, so that it is to be expected that auditory sensitivity should vary roughly as the area of the hearing receptor, and thus about as $M^{2 / 3}$, and this feature is built into the analysis referred to above. While a few animals have narrow-band hearing adapted to detecting particular predators - for example caterpillars detecting wing beats from wasps or for conspecific communication, as in some insects, most higher animals require a wide frequency range so as to detect larger predators, which generally produce sounds of lower frequency, and smaller prey, which may produce higher frequencies. The auditory range for most higher animals therefore extends with reasonable sensitivity over a frequency range of about a factor 300, with the central frequency being higher for smaller animals. In the case of mammals comparable in size to humans, the range for mature adults is typically about $50 \mathrm{~Hz}$ to $15 \mathrm{kHz}$. This wide range, however, means that irrelevant background noise can become a problem, so that conspecific call frequencies may be adapted, even over a short time, to optimize communication. Small echo- 
locating animals, such as bats, generally have a band of enhanced hearing sensitivity and resolution near their call frequency.

Aquatic animals, of course, operate in very different acoustic conditions to land animals, because the density of water nearly matches that of their body tissues. In ad- dition, while the water medium may be considered to be essentially three-dimensional over small distances, it becomes effectively two-dimensional for the very longdistance communication of which some large aquatic animals are capable. Some of these matters will be discussed in Sect. 19.8.

\subsection{Hearing and Sound Production}

Despite the large differences in anatomy and the great separation in evolutionary time between different animals, there are many surprising similarities in their mechanisms of sound production and hearing. The basic mechanism by which sound or vibration is converted to neural sensation is usually one involving displacement of a set of tiny hairs (cilia) mounted on a cell in such a way that their displacement opens an ion channel and causes an electric potential change in the cell, ultimately leading to a nerve impulse. Such sensory hairs occur in the lateral line organs of some species of fish, supporting the otoliths in other aquatic species, and in the cochlea of land-dwelling mammals. Even the sensory cells of insects are only a little different. In the subsequent discussion there will not be space to consider these matters more fully, but they are treated in more detail in Chap. 12.

Somewhat surprisingly, the auditory sensitivities of animals differing widely in both size and anatomy vary less than one might expect. Human hearing, for example, has a threshold of about $20 \mu \mathrm{Pa}$ in its most sensitive frequency range between $500 \mathrm{~Hz}$ and $5 \mathrm{kHz}$, where most auditory information about conspecific communication and the cries of predators and prey is concentrated, and the sensitivity is within about $10 \mathrm{~dB}$ of this value over a frequency range from about $200 \mathrm{~Hz}$ to $7 \mathrm{kHz}$. Other mammals have very similar sensitivities with frequency ranges scaled to vary roughly as the inverse of their linear dimensions, as discussed in Sect. 19.1. Insects in general have narrower bandwidth hearing matched to song frequencies for conspecific communication. Of course, with all of these generalizations there are many outliers with very different hearing abilities.

Sound production mechanisms fall into two categories depending upon whether or not the animal is actively air-breathing. For air-breathing animals there is an internal air reservoir, the volume and pressure of which are under muscular control, so that air can be either inhaled or exhaled. When exhalation is done through some sort of valve with mechanically resonant flaps or

membranes, it can set the valve into vibration, thus producing an oscillatory exhalation of air and so a sound source. In the case of aquatic mammals, which are also air-breathing, it would be wasteful to exhale the air, so that it is instead moved from one reservoir to another through the oscillating valve, the vibration of the thin walls of one of the reservoirs then radiating the sound, as is discussed in more detail in Chap. 19. Animals such as insects that do not breathe actively must generally make use of muscle-driven mechanical vibrations to produce their calls, though a few such as the cockroach Blaberus can actually make "hissing" noises through its respiratory system when alarmed.

The amount of muscular effort an animal is prepared to expend on vocalization varies very widely. Humans, apart from a few exceptions such as trained singers, can produce a maximum sound output of a few hundred milliwatts for a few seconds, and only about $10 \mathrm{~mW}$ for an extended time. In terms of body mass, this amounts to something around $0.1 \mathrm{~mW} \mathrm{~kg}^{-1}$. At the other end of the scale, some species of cicada with body mass of about $1 \mathrm{~g}$ can produce about $1 \mathrm{~mW}$ of sound output at a frequency of about $3 \mathrm{kHz}$, or about $1000 \mathrm{~mW} \mathrm{~kg}^{-1}$ on a nearly continuous basis. An interesting comparative study of birdsongs by Brackenbury [19.15] showed sound powers ranging from 0.15 to $200 \mathrm{~mW}$ and relative powers ranging from 10 to $870 \mathrm{~mW} \mathrm{~kg}^{-1}$. The clear winner on the relative power criterion was the small Turdus philomelos with a sound output of $60 \mathrm{~mW}$ and a body mass of only $69 \mathrm{~g}$, equivalent to $870 \mathrm{~mW} \mathrm{~kg}^{-1}$, while the loudest bird measured was the common rooster $\mathrm{Gal}$ lus domesticus with a sound output during crowing of $200 \mathrm{~mW}$. Its body mass of $3500 \mathrm{~g}$ meant, however, that its relative power was only $57 \mathrm{~mW} \mathrm{~kg}^{-1}$.

Nearly all sound-production mechanisms, ranging from animals through musical instruments to electrically driven loudspeakers, are quite inefficient, with a ratio of radiated acoustic power to input power of not much more than $1 \%$, and often a good deal less than this. In the case of air-breathing animals, the losses 
are largely due to viscosity-induced turbulence above the vocal valve, while in insects that rely upon vibrating body structures internal losses in body tissue are dominant. Wide-bandwidth systems such as the human vocal apparatus are generally much less efficient than narrow-band systems, the parameters of which can be optimized to give good results at a single song frequency. To all this must then be added the internal metabolic loss in muscles, which has not been considered in the $1 \%$ figure.

\subsection{Vibrational Communication}

Most animals are sensitive to vibrations in materials or structures with which they have physical contact. Even humans can sense vibrations over a reasonably large frequency range by simply pressing finger tips against the vibrating object, while vibrations of lower frequency can also be felt through the feet, without requiring any specialized vibration receptors. Many insects make use of this ability to track prey and also for conspecific communication, and some for which this mode of perception is very important have specialized receptors, called subgenual organs, just below a leg joint. A brief survey for the case of insects has been given by Ewing [19.16].

For water gliders and other insects that hunt insects that have become trapped by surface tension on the surface of relatively still ponds, the obvious vibratory signal is the surface waves spreading out circularly from the struggles of the trapped insect. The propagation of these surface waves on water that is deep compared with the wavelength has been the subject of analysis, and the calculated wave velocity $c_{\mathrm{s}}$, which is influenced by both the surface tension $T$ of water and the gravitational acceleration $g$, is found to depend upon the wavelength $\lambda$ according to the relation

$$
c_{\mathrm{s}}=\left(\frac{2 \pi T}{\rho_{\mathrm{w}} \lambda}+\frac{g \lambda}{2 \pi}\right)^{1 / 2},
$$

where $\rho_{\mathrm{w}}$ is the density of water. From the form of this relation it is clear that the wave speed has a minimum value when $\lambda=2 \pi\left(T / g \rho_{\mathrm{w}}\right)^{1 / 2}$, and substituting numerical values then gives a wavelength of about $18 \mathrm{~mm}$, a propagation speed of about $24 \mathrm{~cm} \mathrm{~s}^{-1}$, and a frequency of about $1.3 \mathrm{~Hz}$. Waves of about this wavelength will continue to move slowly across the surface after

\subsection{Insects}

The variety of sound production and detection mechanisms across insect species is immense [19.3,16-18], but their acoustic abilities and senses possess many features in common. Many, particularly immature forms such as caterpillars but also many other insects, use external sen-

other waves have dissipated. The attenuation of all these surface waves is, however, very great.

From (19.1) it is clear that, at low frequencies and long wavelengths, the wave speed $c_{\mathrm{s}}$ is controlled by gravitational forces and $c_{\mathrm{s}} \approx g / \omega$. These are called gravity waves. At high frequencies and short wavelengths, surface tension effects are dominant and $c_{\mathrm{s}} \approx\left(T \omega / \rho_{\mathrm{w}}\right)^{1 / 3}$. These are called capillary waves. The frequencies of biological interest are typically in the range 10 to $100 \mathrm{~Hz}$, and so are in the in the lower part of the capillary-wave regime, a little above the crossover between these two influences, and the propagation speeds are typically in the range 30 to $50 \mathrm{~cm} \mathrm{~s}^{-1}$.

For insects and other animals living aloft in trees and plants, the waves responsible for transmission of vibration are mostly bending waves in the leaves or branches. The wave speed is then proportional to the square root of the thickness of the structure and varies also as the square root of frequency. Transmission is very slow compared with the speed of sound in air, although generally much faster than the speed of surface waves on water. Once again, the attenuation with propagation distance is usually large.

Disturbances on heavy solid branches or on solid ground also generate surface waves that can be detected at a distance, but these waves propagate with nearly the speed of shear waves in the solid, and thus at speeds of order $2000 \mathrm{~m} \mathrm{~s}^{-1}$. On the ground they are closely related to seismic waves, which generally have much larger amplitude, and animals can also detect these. Underground, of course, as when an animal is seeking prey in a shallow burrow, the vibration is propagated by bulk shear and compressional waves.

sory hairs to detect air movement and these can sense both acoustic stimuli, particularly if tuned to resonance, or the larger near-field air flows produced by the wings of predators [19.19]. These hairs respond to the viscous drag created by acoustic air flow past them and, since 
this is directional, the insect may be able to gain some information about the location of the sound source.

Analysis of the behavior of sensory hairs is complex [19.19], but some general statements can be made. Since what is being sensed is an oscillatory flow of air across the surface of the insect, with the hair standing normal to this surface, it is important that the hair be long enough to protrude above the viscous boundary layer, the thickness of which varies inversely with the square root of frequency and is about $0.1 \mathrm{~mm}$ at $150 \mathrm{~Hz}$. This means that the length of the hair should also be about inversely proportional to the frequency of the stimulus it is optimized to detect, and that it should typically be at least a few tenths of a millimeter in length. The thickness of the hair is not of immense importance, provided that it is less than about $10 \mu \mathrm{m}$ for a typical hair, since much of the effective mass is contributed by co-moving fluid. At the mechanical resonance frequency of the hair, the sensitivity is a maximum, and the hair tip displacement is typically about twice the fluid displacement, provided the damping at the hair root is not

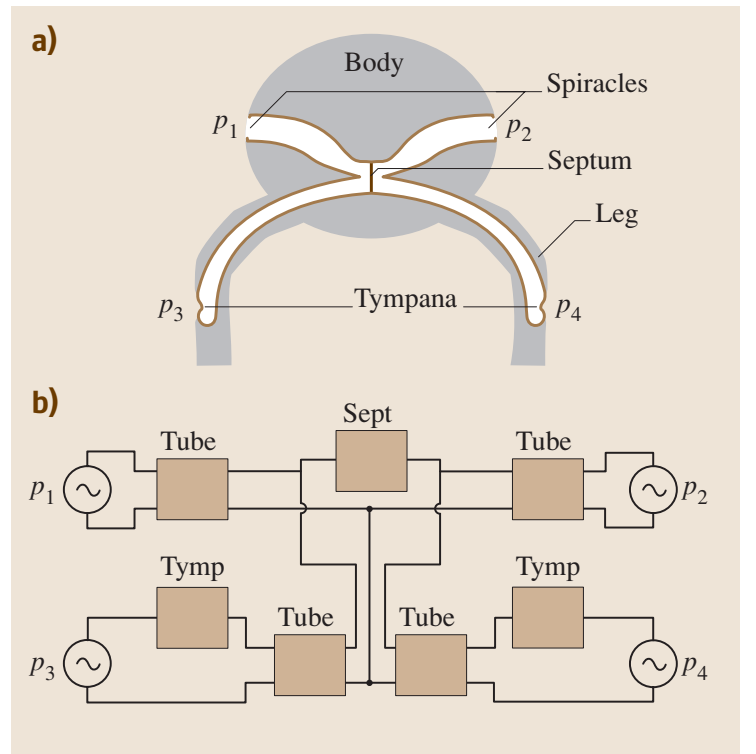

Fig. 19.2 (a) Schematic drawing of the auditory anatomy of a cricket. (b) Simplified electric network analog for this system. The impedances of the tympana and the septum are each represented by a set of components $L, R, C$ connected in series; the tube impedances are represented by $2 \times 2$ matrices. The acoustic pressures $p_{i}$ acting on the system are generally all nearly equal in magnitude, but differ in phase depending upon the direction of incidence of the sound large, but the response falls off above and below this frequency.

Mature insects of some species, however, have auditory systems that bear a superficial resemblance to those of modern humans in their overall structure. Thus for example, and referring to Fig. 19.2a, the cricket has thin sensory membranes (tympana or eardrums) on its forelegs, and these are connected by tubes (Eustachian tubes) that run to spiracles (nostrils) on its upper body, the left and right tubes interacting through a thin membrane (nasal septum) inside the body. Analysis of this system [19.9] shows that the tubes and membranes are configured so that each ear has almost cardioid response, directed ipsilaterally, at the song frequency of the insect, allowing the insect to detect the direction of sound arrival. Some simpler auditory systems showing similar directional response are discussed in Sect. 19.5.

Detailed analysis of the response of anatomically complex systems such as this is most easily carried out using electric network analogs, in which pressure is represented by electric potential and acoustic flow by electric current. Tubes are then represented as $2 \times 2 \mathrm{ma-}$ trices, diaphragms by $L, R, C$ series resonant circuits, and so on, as shown in Fig. 19.2b. Brief details of this approach are given in Sect. 19.10 and fuller treatments can be found in the published literature $[19.9,20]$.

When it comes to sound production, insects are very different from other animals because they do not have the equivalent of lungs and a muscle-driven respiratory systems. They must therefore generally rely upon muscle-driven vibration of diaphragms somewhere on the body in order to produce sound. One significant exception is the Death's Head Hawk Moth Acherontia atropos, which takes in and then expels pulses of air from a cavity closed by a small flap-valve that is set into oscillation by the expelled air to produce short sound pulses [19.16].

Some insects, such as cicadas, have a large abdominal air cavity with two areas of flexible ribbed cartilage that can be made to vibrate in a pulsatory manner under muscle action, progressive buckling of the membrane as the ribs flip between different shapes effectively multiplying the frequency of excitation by as much as a factor 50 compared with the frequency of muscle contraction. The coupling between these tymbal membranes and the air cavity determines the oscillation frequency, and radiation is efficient because of the monopole nature of the source. The sound frequency is typically in the range 3 to $5 \mathrm{kHz}$, depending upon insect size, and the radiated acoustic power can be as large as $1 \mathrm{~mW}$ [19.21] on an almost continuous basis, though the 


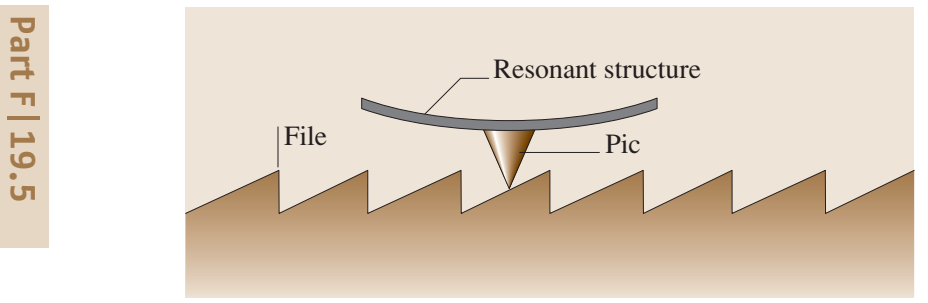

Fig. 19.3 Configuration of a typical file and pic as used by crickets and other animals to excite resonant structures on their wing cases

actual sound signal may consist of a series of regularly spaced bursts corresponding to tymbal collapses and rebounds. Again, there are occasional insects that have developed extreme versions of this sound producing mechanism, such as the bladder cicada Cystosoma Saundersii (Westwood), in which the whole greatly extended abdomen is a resonant air sac and the song frequency is only $800 \mathrm{~Hz}$.

Most other insects do not have significant bodily air cavities and must produce sound by causing parts of their body shell or wings to vibrate by drawing a ribbed file on their legs, or in some cases on their wings, across the hard rim of the membrane, as shown in Fig. 19.3. The anatomical features making up the file and vibrating structure vary very greatly across species, as has been discussed and illustrated in detail by Dumortier [19.22]. In some cases the file is located on the vibrating structure and the sharp pic on the part that is moving. The passage of the pic across each tooth or rib on the file generates a sharp impulse that excites a heavily damped transient oscillation of the vibrator, and each repetitive motion of the leg or other structure passes the pic over many teeth. A typical insect call can therefore be subdivided into (a) the leg motion frequency, (b) the pic impact frequency on the file, and (c) the oscillation frequency of the vibrating structure. Since the file may have 10 or more teeth, the frequency of the pic impacts will be more than 10 times the frequency of leg motion, and

\subsection{Land Vertebrates}

The class of vertebrates that live on land is in many ways the most largely studied, since they bear the closest relation to humans, but these animals vary widely in size and behavior. Detailed discussion of birds is deferred to Sect. 19.6, since their songs warrant special attention, while human auditory and vocal abilities are not con-
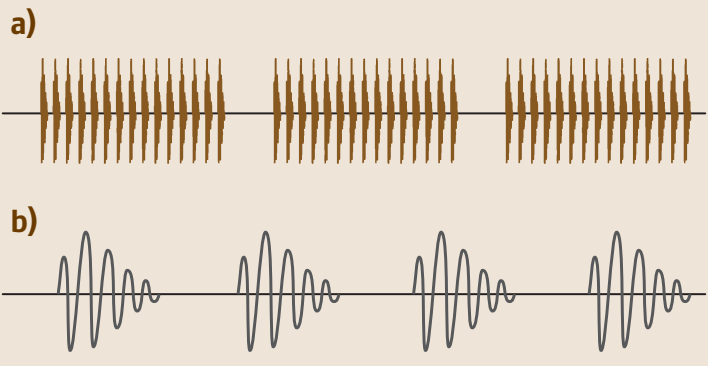

Fig. 19.4a,b Structure of the sound emitted by a typical insect: (a) three strokes of the file, the individual oscillations being the pic impacts on the file teeth; (b) expanded view of four of the pulses in (a), showing resonant oscillations of the structure produced by successive pic impacts on the file

the structural oscillation may be 10 times this frequency. The structure of a typical call is thus of the form shown in Fig. 19.4. The dominant frequency is usually that of the structural vibration, and this varies widely from about 2 to $80 \mathrm{kHz}$ depending upon species and size [19.23]. Because of the structure of the call, the frequency spectrum appears as a broad band centered on the structural vibration frequency.

Such a vibrating membrane is a dipole source and so is a much less efficient radiator than is a monopole source, except at very high frequencies where the wing dimensions become comparable with the sound wavelength excited. Small insects therefore generally have songs of higher frequency than those that are larger, in much the same way as discussed in Sect. 19.1 for other animals. Some crickets, however, have evolved the strategy of digging a horn-shaped burrow in the earth and positioning themselves at an appropriate place in the horn so as to couple their wing vibrations efficiently to the fundamental mode of the horn resonator, thus taking advantage of the dipole nature of their wing source and greatly enhancing the radiated sound power [19.24].

sidered specifically since they are the subject of several other chapters. Reptiles, too, are largely omitted from detailed discussion, since most of them are not notably vocal, and bats are given special attention in Sect. 19.7.

The feature possessed by all the land-dwelling vertebrates is that they vocalize using air expelled from their 
inflated lungs through some sort of oscillating vocal valve. The available internal air pressure depends upon the emphasis put by the species on vocalization, but is typically in the range $100-2000 \mathrm{~Pa}$ (or $1-20 \mathrm{~cm}$ water gauge) almost independent of animal size, which is what is to be expected if the breathing muscles and lung cavity diameter scale about proportionally with the linear size of the animal. There are, however, very wide variations, as discussed in Sect. 19.2. The auditory abilities of most animals are also rather similar, the $20 \mathrm{~dB}$ bandwidth extending over a factor of about 100 in frequency and with a center-frequency roughly inversely proportional to linear size, though again there are outliers, as shown in Fig. 19.1. Although the auditory and vocalization frequencies of different animals both follow the same trend with size, the auditory systems generally have a much larger frequency range than the vocalizations for several reasons. The first is that hearing serves many purposes in addition to conspecific communication, particularly the detection of prey and predators. The second is that, particularly with the more sophisticated animals, there are many nuances of communication, such as the vowels and consonants in human speech, for which information is coded in the upper parts of the vocalization spectrum.

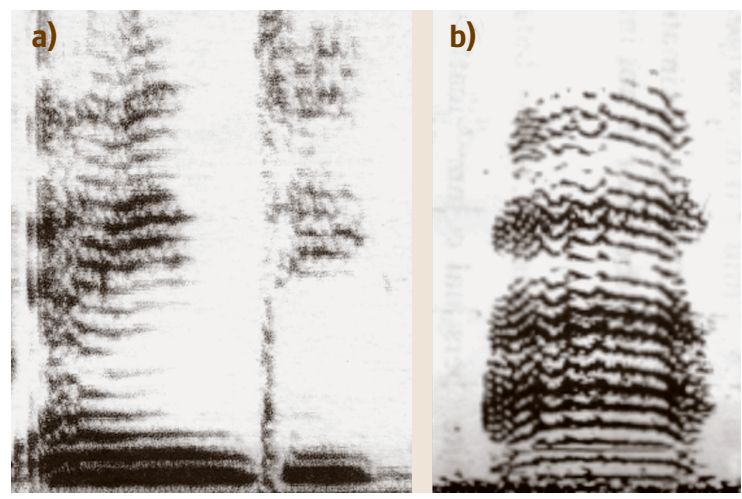

Fig. 19.5a,b A typical time-resolved spectrum for (a) human female speech, and (b) the cry of a raven, the level of each frequency component being indicated by its darkness. The frequency range is $0-6 \mathrm{kHz}$ and the duration about $0.7 \mathrm{~s}$ in (a) and $0.5 \mathrm{~s}$ in (b). It can be seen that at any instant the spectrum consists of harmonics of the fundamental, with particular bands of frequencies being emphasized. These are the formant bands that distinguish one vowel from another in humans and enable similar distinctions to be made by other animals. Consonants in human speech consist of broadband noise, an example occurring at the beginning of the second syllable in (a), and other animals may have similar interpolations between the tonal sounds
For humans, for example, the primary vocalization frequency is typically in the range 100 to $300 \mathrm{~Hz}$, but the frequency bands that are emphasized in vowel formants lie between 1 and $3 \mathrm{kHz}$. The same pattern is exhibited in the vocalizations of other air-breathing animals, as shown for the case of a raven in Fig. 19.5. The same formant structure is seen in the cries of elephants, with a fundamental in the range $25-30 \mathrm{~Hz}$ and in high-pitched bird songs, which may have a fundamental above $4 \mathrm{kHz}$.

In most such animals, sound is produced by exhaling the air stored in the lungs through a valve consisting of two taut tissue flaps or membranes that can be made to almost close the base of the vocal tract, as shown in Fig. 19.6a. The lungs in mammals are a complicated

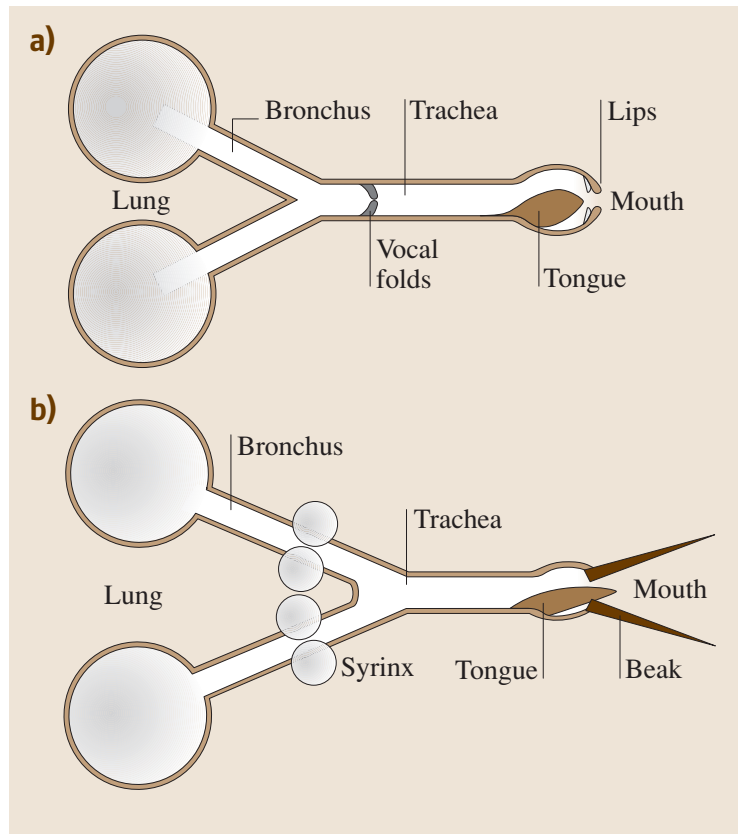

Fig. 19.6 (a) Sketch of the vocal system of a typical land mammal. The lungs force air through the dual-flap vocalfold valve, producing a pulsating flow of air that is rich in harmonics of the fundamental frequency. Resonances of the upper vocal tract, which can be modified by motion of the tongue and lips, produce formants in the sound spectrum which encode vocal information. (b) Sketch of the vocal system of a typical song-bird. There are two inflatedmembrane valves in the syrinx just below the junction of the bronchi with the trachea, which may be operated either separately or together. Again a harmonic-rich airflow is produced that can either have formants imposed by resonances of the vocal tract and beak, or can be filtered to near pure-tone form by an inflated sac in the upper vocal tract 
quasi-fractal dendritic network of tubules with as many as 16 stages of subdivision, the final stage being terminated by alveolar sacs that provide most of storage volume. The interaction of exhaled air pressure and air flow with the flaps of this valve when they have been brought together (or adducted) causes them to vibrate at very nearly their natural frequency, as determined by mass and tension, and this in turn leads to an oscillating air flow through the valve. The classic treatment of the human vocal valve is that of Ishizaka and Flanagan [19.25], but there have been many more recent treatments exploring modifications and refinements of this model. Very similar vocal valves are found in other mammals, while the major difference in the case of birds is that the flaps of the vocal folds are replaced by opposed taut membranes inflated by air pressure in cavities behind them, and there may be two such valves, as shown in Fig. 19.6b and discussed later in Sect. 19.6.

In most cases, the vibration frequency of the valve is very much lower than the frequency of the first maximum in the upper vocal tract impedance. The valve therefore operates in a nearly autonomous manner at a frequency determined by its geometry, mass and tension and, to a less extent, by the air pressure in the animal's lungs. Because any such valve is necessarily nonlinear in its flow properties, particularly if it actually closes once in each oscillation cycle, this mechanism generates an air flow, and thus a sound wave in the vocal tract, containing all harmonics of the fundamental oscillation. The radiated amplitudes of these harmonics can be modified by resonances in the air column of the vocal tract to produce variations in sound quality and peaks in the radiated spectrum known generally as vocal formants. Changing the tension of the valve flaps changes the fundamental frequency of the vocal sound, but the frequencies of the formants can be changed independently by moving the tongue, jaws, and lips or beak.

To be more quantitative, suppose that the pressure below the valve is $p_{0}$ and that above the valve $p_{1}$. If the width of the opening is $W$ and its oscillating dimension is $x(t)$, then the volume flow $U_{1}$ through it is determined primarily by Bernoulli's equation and is approximately

$$
U_{1}(t)=\left(\frac{2\left(p_{0}-p_{1}\right)}{\rho}\right)^{1 / 2} W x(t),
$$

where $\rho$ is the density of air. In order for the valve to be maintained in oscillation near its natural frequency $f$, the pressure difference $p_{0}-p_{1}$ must vary with the flow and with a phase that is about $90^{\circ}$ in advance of the valve opening $x(t)$. This can be achieved if the acous- tic impedance of the lungs and bronchi is essentially compliant, and that of the upper vocal tract small, at the oscillation frequency. The fact that this pressure difference appears as a square root in (19.2) then introduces upper harmonic terms at frequencies $2 f, 3 f, \ldots$ into the flow $U(t)$. Allowance must then be made for the fact that the vocal valve normally closes for part of each oscillation cycle, so that $x(t)$ is no longer simply sinusoidal, and this introduces further upper-harmonic terms into the flow. This is, of course, a very condensed and simplified treatment of the vocal flow dynamics, and further details can be found in the literature for the case of mammalian, and specifically human, animals [19.25] and also for birds [19.26].

This is, however, only the beginning of the analysis, for it is the acoustic flow out of the mouth that determines the radiated sound, rather than the flow from the vocal valve into the upper vocal tract. Suppose that the acoustic behavior of the upper vocal tract is represented by a $2 \times 2$ matrix, as shown in Fig. 19.11 of Sect. 19.10, and that the mouth or beak is regarded as effectively open so that the acoustic pressure $p_{2}$ at this end of the tract is almost zero. Then the acoustic volume flow $U_{2}$ out of the mouth at a particular angular frequency $\omega$ is

$$
U_{2}=\frac{Z_{21}}{Z_{22}} U_{1},
$$

where $U_{1}$ is the flow through the larynx or syrinx at this frequency. For the case of a simple cylindrical tube of length $L$ at angular frequency $\omega$, this gives

$$
U_{2}=\frac{U_{1}}{\cos k L},
$$

where $k=(\omega / c)-\mathrm{i} \alpha, c$ is the speed of sound in air, and $\alpha$ is the wall damping in the vocal tract. If the spectrum of the valve flow $U_{1}$ has a simple envelope, as is normally the case, then (19.4) shows that the radiated sound power, which is proportional to $\omega^{2} U_{2}^{2}$, has maxima when $\omega L / c=(2 n-1) \pi / 2$, and thus in a sequence $1,3,5, \ldots$ These are the formant bands, the precise frequency relationship of which can be varied by changing the geometry of the upper vocal tract, which in turn changes the forms of $Z_{21}$ and $Z_{22}$.

Some other air-breathing animals (and even human sopranos singing in their highest register) however, adjust their vocal system so that the frequency of the vocal valve closely matches a major resonance of the upper vocal tract, usually that of lowest frequency but not necessarily so. Some species of frogs and birds achieve this by the incorporation of an inflatable sac in the upper vocal tract. In some cases the sac serves simply to provide 
a resonance of appropriate frequency, and the sound is still radiated through the open mouth, but in others the walls of the sac are very thin so that they vibrate under the acoustic pressure and provide the primary radiation source. This will be discussed again in Sect. 19.6 in relation to bird song, but the analysis for frog calls is essentially the same.

The overall efficiency of sound production in vertebrates is generally only $0.1-1 \%$, which is about the same as for musical instruments. The acoustic output power output is typically in the milliwatt range even for quite large animals, but there is a very large variation between different species in the amount of effort that is expended in vocalization.

The hearing mechanism of land-dwelling vertebrates shows considerable similarity across very many species. Sound is detected through the vibrations it induces in a light taut membrane or tympanum, and these vibrations are then communicated to a transduction organ where they are converted to nerve impulses, generally through the agency of hair cells. The mechanism of neural transduction and frequency discrimination is complex, and its explanation goes back to the days of Helmholtz [19.27] in the 19th century, with the generally accepted current interpretation being that of von Békésy [19.28] in the mid-20th century. Although these studies related specifically to human hearing, the models are generally applicable to other land-dwelling vertebrates as well, as surveyed in the volume edited by Lewis et al. [19.29]. All common animals have two auditory transducers, or ears, located on opposite sides of the head, so that they are able to gain information about the direction from which the sound is coming.

In the simplest such auditory system, found for example in frogs, the two ears open directly into the mouth cavity, as shown in Fig. 19.7a, one neural transducer being closely coupled to each of the two tympana. Such a system is very easily analyzed at low frequencies where the cavity presents just a simple compliance with no resonances of its own. Details of the approach are similar to those for the more complex system to be discussed next. For a typical case in which the ears are separated by $20 \mathrm{~mm}$, the cavity volume is $1 \mathrm{~cm}^{3}$, and the loaded tympanum resonance is at $500 \mathrm{~Hz}$, the calculated response has the form shown in Fig. 19.7b. Directional discrimination is best at the tympanum resonance frequency and can be as much as $20 \mathrm{~dB}$. In a more realistic model for the case of a frog, the nostrils must also be included, since they lead directly into the mouth and allow the entry of sound. The calculated results in this case show that the direction of maximum response for each ear is

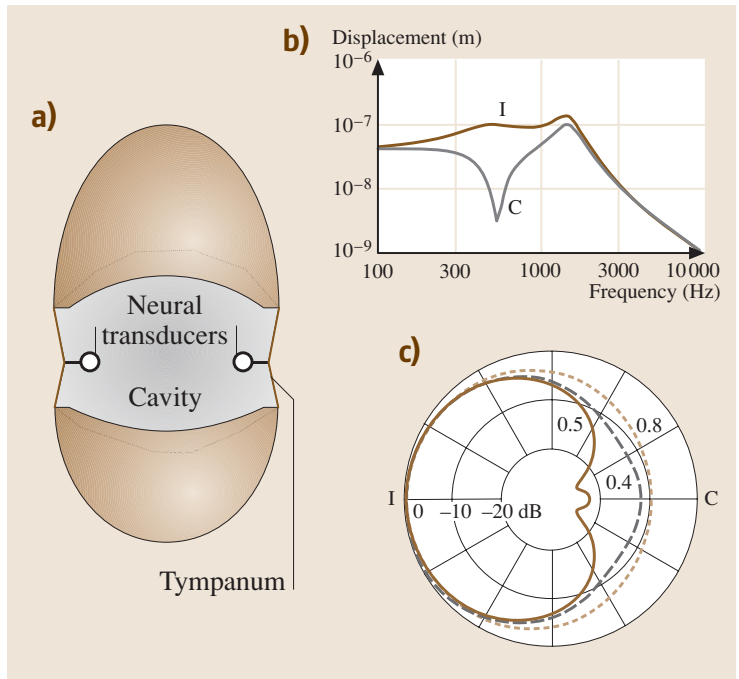

Fig. 19.7 (a) A simple frog-ear model; (b) the calculated frequency response for ipsilateral (I) and contralateral (C) sound signals for a particular optimized set of parameter values; (c) the calculated directivity at different frequencies, shown in $\mathrm{kHz}$ as a parameter (from [19.9])

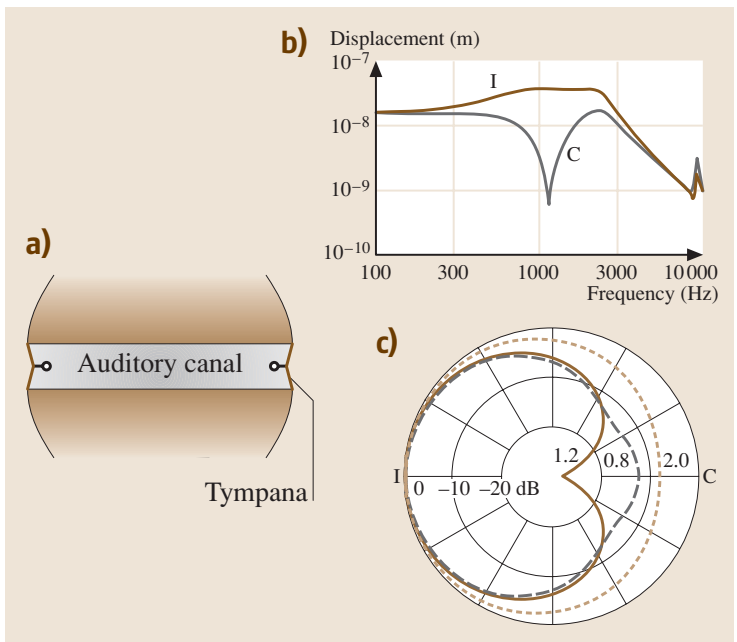

Fig. 19.8 (a) Idealized model of the auditory system of a lizard or bird. Each tympanum is connected to a neural transducer and the two are joined by a simple tube. (b) Response of a particular case of the system in (a) to ipsilateral (I) and contralateral (C) sound of amplitude $0.1 \mathrm{~Pa}$ (equivalent to $74 \mathrm{~dB}$ re $20 \mu \mathrm{Pa}$ ). The tube diameter is assumed to be $5 \mathrm{~mm}$ and its length $20 \mathrm{~mm}$, while the tympana have a thickness of $10 \mu \mathrm{m}$ and are tensioned to a resonance frequency of $1000 \mathrm{~Hz}$. (c) Directional response of this hearing system (from [19.9]) 
shifted towards the rear of the animal, typically by as much as $30^{\circ}$ [19.9].

In another simple auditory system such as that of most reptiles, and surprisingly birds, the two tympana are located at the ends of a tube passing through the head and each is supplied with its own neural transducer, as shown in Fig. 19.8a. The tympana may be recessed at the end of short tubes to provide protection, these tubes only slightly modifying the frequency response. The behavior of such a system can be analyzed using the electric network analogs discussed in Sect. 19.10, with each tympanum involving a series $L, R, C$ combination and the tube being represented by a $2 \times 2$ impedance matrix $Z_{i j}$. If the sound comes from straight in front, then it is the same in magnitude and phase at each ear, so that their responses are necessarily the same. When the sound comes from one side, however, there is a phase difference between the two ear pressures, along with a rather less significant amplitude difference. The motion of each tympanum is determined by the difference between the internal and external pressures acting upon it, and the internal pressure depends upon the signal transferred from the opposite tympanum, modified by the phase delay or resonance of the coupling tube. The analysis is straightforward, but the resulting behavior, which depends upon the mass and tension of the tympana and the length and diameter of the connecting tube, can only be determined by explicit calculation [19.9]. When these parameters are appropriately chosen, each ear has a strongly directional response near the resonance frequency of the tympana, as shown in Fig. 19.8b, c. The balance between the depth and sharpness of the contralateral minimum is affected by the acoustic absorption in the walls of the connecting canal. In some animals, the bone lining of this canal is actually porous, which reduces the effective sound-wave velocity inside it and so increases the phase shift between internal and external signals, a feature that is of assistance when the physical separation of the ears is small.

In most mammals and other large animals, the auditory system is modified in several significant ways. The first is that the auditory canal joining the two ears in birds and reptiles has generally degenerated in mammals to the extent that each ear functions nearly independently. The connecting canal in humans has become the pair of Eustachian tubes running from the middle ear cavity, which contains the bones linking the tympanum to the cochlea, down to the nasal cavities, and its main purpose is now simply to equalize internal and external static pressures and to drain excess fluids. The middle ear cavity itself is necessary in order that the enclosed air volume be large enough that it does not raise the resonance frequency of the tympanum by too much. The topology of the whole system is surprisingly similar to that of the cricket auditory system shown in Fig. 19.2a, but the functions of some of the elements are now rather different.

The other major change is that, instead of the tympana being located almost on the surface of the animal, they are buried below the surface at the end of short auditory canals (meati), which lead to external ears (pinnae) in the shape of obliquely truncated horns. As well as protecting the tympana from mechanical damage, the canals add a minor resonance of their own, generally in the upper part of the auditory range of the animal concerned. The pinnae both increase the level of the pressure signal, typically by about $10 \mathrm{~dB}$ and in some cases even more, and impart a directionality to the response $[19.9,30]$. The convoluted form of some pinnae also imparts directionally-excited resonances that help distinguish direction from tonal cues, a feature that is necessary in order to distinguish up/down directions for sounds coming from immediately ahead or immediately behind. Some animals with particularly large pinnae, such as kangaroos, are able to rotate these independently to help locate a sound source. In all such cases, however, the neural system plays a large part in determining sound direction by comparing the phases and amplitudes, and sometimes the precise timing of transients, in the signals received by the two ears.

In a hearing system of any simple type it is clear that, if geometrical similarity is maintained and the system is simply scaled to the linear size of the animal concerned, then the frequency of maximum discrimination will vary as the inverse of the linear size of the animal, giving an approximate match to general trend of vocalization behavior. There is, however, one anomaly to be noted [19.31]. Vocalizations by some frogs have fundamental frequencies as high as $8 \mathrm{kHz}$ and the vocal signal contains prominent formant bands in the case studied at $20 \mathrm{kHz}$ and $80 \mathrm{kHz}$, so that they might almost be classed as ultrasonic. Despite this, behavioral evidence indicates that these frogs cannot hear signals much above $10 \mathrm{kHz}$, so that perhaps the formant bands are simply an epiphenomenon of the sound production system. The same is true of the calls of some birds. 


\subsection{Birds}

Because of their generally melodious songs, birds have excited a great deal of attention among researchers, as described in several classic books [19.32,33]. Hearing does not require further discussion because the auditory system is of the simple tube-coupled type discussed in Sect. 19.5 and illustrated in Fig. 19.8. Research interest has focused instead on the wide range of song types that are produced by different species. These range from almost pure-tone single-frequency calls produced by doves, and whistle-like sounds sweeping over more than a 2:1 frequency range produced by many other species, through wide-band calls with prominent formants, to the loud and apparently chaotic calls of the Australian sulfur-crested cockatoo. Some birds can even produce two-toned calls by using both syringeal valves simultaneously but with different tuning.

As noted in Sect. 19.5 and illustrated in Fig. 19.6b, song birds have a syrinx consisting of dual inflatedmembrane valves, one in each bronchus just below their junction with the trachea. These valves can be operated simultaneously and sometimes at different frequencies, but more usually separately, and produce a pulsating air-flow rich in harmonics. In birds such as ravens, the impedance maxima of the vocal tract, as measured at the syringeal valve, produce emphasized spectral bands or formants much as in human speech, as is shown in Fig. 19.5. The vocal tract of a bird is much less flexible in shape than is that of a mammal, but the formant frequencies can still be altered by changes in tongue position and beak opening. Studies with laboratory models [19.34] show that the beak has several acoustic functions. When the beak is nearly closed, it presents an end-correction to the trachea that is about half the beak length at high frequencies, but only about half this amount at low frequencies, so that the formant resonances are brought closer together in frequency. As the beak gape is widened, the end-correction reduces towards the normal value for a simple open tube at all frequencies. The beak also improves radiation efficiency, particularly at higher frequencies, by about $10 \mathrm{~dB}$ for the typical beak modeled. Finally, the beak enhances the directionality of the radiated sound, particularly at high frequencies and for wide gapes, this enhanced directionality being as much as $10 \mathrm{~dB}$ compared with an isotropic radiator.

The role of the tongue has received little attention as yet, partly because it is rather stiff in most species of birds. It does, however, tend to constrict the passage between the top of the trachea and the root of the beak, and this constriction can be altered simply by raising the tongue, thereby exaggerating the end-correction provided to the trachea by the beak. These models of beak behavior can be combined with models of the syrinx and upper vocal tract [19.26] to produce a fairly good understanding of vocalization in birds such as ravens. The fundamental frequency of the song can be changed by changing the muscle tension and thus the natural resonance frequency of the syringeal valve, while the formants, and thus the tone of the song, can be varied by changing the beak opening and tongue position. The role of particular anatomical features and muscular activities in controlling the fundamental pitch of the song has since been verified by careful measurement and modeling [19.35,36].

Some birds have developed the ability to mimic others around them, a notable example being Australian Lyrebirds of the family Menuridae which, as well as imitating other birds, have even learned to mimic humangenerated sounds such as axe blows, train whistles, and even the sound of film being wound in cameras. There has also been considerable interest in the vocalization of certain parrots and cockatoos, which can produce, as well as melodious songs, quite intelligible imitations of human speech. The important thing here is to produce a sound with large harmonic content and then to tune the vocal tract resonances, particularly those that have frequencies in the range $1-2.5 \mathrm{kHz}$, to match those of the second and third resonances of the human vocal tract, which are responsible for differentiating vowel sounds. Consonants, of course, are wide-band noise-like transients. Careful studies of various parrots [19.37,38] show that they can achieve this by careful positioning of the tongue, much in the way that humans achieve the same thing but with quantitative differences because of different vocal tract size. The match to human vowels in the second and third human formants can be quite good. The first formant, around $500 \mathrm{~Hz}$ in humans, is not reproduced but this is not important for vowel recognition.

These studies produce a good understanding of the vocalization of normal song-birds, the calls of which consist of a dominant fundamental accompanied by a range of upper harmonics. Less work has been done on understanding the nearly pure-tone cries of other birds. For many years there has been the supposition that the sound production mechanism in this case was quite different, perhaps involving some sort of aerodynamic whistle rather than a syringeal vibration. More recent experimental observations on actual singing birds, using 
modern technology [19.39], have established however that, at least in the cases studied, the syringeal valve is in fact normally active, though it does not close completely in any part of the cycle, thus avoiding the generation of large amplitudes of upper harmonics. Suppression of upper harmonics can also be aided by filtering in the upper vocal tract [19.40].

In the case of doves, which produce a brief pure-tone "coo" sound at a single frequency, the explanation appears to lie in the use of a thin-walled inflated esophageal sac and a closed beak, with fine tuning provided by an esophageal constriction [19.41]. The volume $V$ of air enclosed in the sac provides an acoustic compliance $C=V / \rho c^{2}$ that is in parallel with an acoustic inertance $L=m / S^{2}$ provided by the mass $m$ and surface area $S$ of the sac walls. The resonance frequency $f$ of this sac filter is given by

$$
f=\frac{1}{2 \pi}\left(\frac{\rho c^{2} S^{2}}{m V}\right)^{1 / 2},
$$

where $\rho$ is the density of air and $c$ is the speed of sound in air. When excited at this frequency by an inflow of air from the trachea, the very thin expanded walls of the sac vibrate and radiate sound to the surrounding air. For a typical dove using this strategy, the wall-mass and dimensions of the inflated sac are about right to couple to the "coo" frequency of about $900 \mathrm{~Hz}$. From (19.5), the resonance frequency varies only as the square root of the diameter of the sac, so that a moderate exhalation of breath can be accommodated without much disturbance to the resonance. The dove must, however, learn to adjust the glottal constriction to couple the tracheal resonance efficiently to that of the sac.

\subsection{Bats}

Since human hearing, even in young people, is limited to the range from about $20 \mathrm{~Hz}$ to about $20 \mathrm{kHz}$, frequencies lying outside this range are referred to as either infrasonic or ultrasonic. Very large animals such as elephants may produce sounds with appreciable infrasonic components, though the higher harmonics of these sounds are quite audible. Small animals such as bats, however, produce echo-location calls with dominant frequencies around $80 \mathrm{kHz}$ that are inaudible to humans, though some associated components of lower frequency may be heard. In this section brief attention will be given to the sonar abilities of bats, though some other animals also make use of ultrasonic techniques [19.43].
Some other birds, such as the Cardinal, that produce nearly pure-tone calls over a wide frequency range, appear to do so with the help of an inflated sac in the upper vocal tract that leads to the opened beak, with the tracheal length, sac volume, glottal constriction, tongue position, and beak opening all contributing to determine the variable resonance frequency of the filter. Because the sac is not exposed as in the dove, its walls are heavy and do not vibrate significantly. It thus acts as a Helmholtz resonator, excited by the input flow from the trachea and vented through the beak. The bird presumably learns to adjust the anatomical variables mentioned above to the syringeal vibration frequency, which is in turn controlled by syringeal membrane tension, and can then produce a nearly pure-tone song over quite a large frequency range. Despite this explanation of the mechanism of pure-tone generation in some cases, others have still to be understood, for the variety of birdsong is so great that variant anatomies and vocalization skills may well have evolved.

With most of the basic acoustic principles of birdsong understood, most of the interest in birdsong centers upon the individual differences between species. The variety found in nature is extremely great and, while sometimes correlated with large variations in bird anatomy, it also has many environmental influences. Some birds, surprisingly, have songs with prominent formant bands extending well into the ultrasonic region, despite the fact that behavioral studies and even auditory brainstem measurements show that they have no response above about $8 \mathrm{kHz}[19.31,42]$. The formant bands in these cases are therefore presumably just an incidental product of the vocalization mechanism.

Sound waves are scattered by any inhomogeneities in the medium through which they are propagating, the density of an animal body in air provides a large contrast in acoustic impedance and therefore a large scattered signal. The basic physics shows that, for a target in the form of a sphere of diameter $d$ and a signal of wavelength $\lambda \gg d$, the echo strength is proportional to $d^{6} / \lambda^{4}$ times the incident intensity. This incident intensity is itself determined by the initial signal power and the angular width of the scanning signal, which varies about as $\lambda / a$, where $a$ is the radius of the mouth of the probing animal. If $R$ is the distance between the probing animal and the target, then the sound power incident on the target varies as $a^{2} / \lambda^{2} R^{2}$ and the returned echo strength $E$ thus varies 
as

$$
E \propto \frac{d^{6} a^{2}}{\lambda^{6} R^{4}},
$$

provided the wavelength $\lambda$ is much greater than the diameter $d$ of the scattering object and the radius $a$ of the mouth. Another factor must, however, be added to this equation and that is the attenuation of the sound due to atmospheric absorption. This attenuation depends on relative humidity, but is about $0.1 \mathrm{~dB} \mathrm{~m}^{-1}$ at $10 \mathrm{kHz}$, increasing to about $0.3 \mathrm{~dB} \mathrm{~m}^{-1}$ at $100 \mathrm{kHz}$, and so is not a very significant factor at the short ranges involved.

Once the size of the target becomes comparable with the incident sound wavelength, the scattering tends towards becoming reflection and geometrical-optics techniques can be used. In this limit, the reflected intensity is proportional to the area of the target, but depends in a complicated way upon its shape and orientation. Finally, if a pure-tone sound is used, any Doppler shift in the frequency of the echo can give information about the relative motion of prey and predator. The reflected sound can therefore give information about the nature of the target and its motion, both of which are important to the pursuing bat.

The sonar-location cries of bats and other animals are, however, not pure-tone continuous signals but rather sound pulses with a typical frequency in the range $40-80 \mathrm{kHz}$ and so a wavelength of about $4-8 \mathrm{~mm}$. This wavelength is comparable to the size of typical prey insects so that the echo can give information about size, shape and wing motion, while the echo delay reveals the distance. The bat's large ears are also quite directional at these frequencies, so that further information on location is obtained. Rather than using short bursts of pure tone, some predators use longer bursts in which the frequency is swept rapidly through a range of several

\subsection{Aquatic Animals}

The generation and propagation of sound under water, as discussed in Chap. 19, have many features that are different from those applying to sound in air. From a physical point of view, a major difference is the fact that water is often quite shallow compared with the range of sound, so that propagation tends to be two-dimensional at long distances. From the present biological viewpoint the major difference is that the acoustic impedance of biological tissue is nearly equal to that of the surrounding water, while in air these differ by a factor of about kilohertz. This technique is known as "chirping", and it allows the returned echo to be reconstructed as a single pulse by frequency-dependent time delay, although animal neural systems may not actually do this. The advantage of chirping is that the total energy contained in the probing pulse, and thus the echo, can be made much larger without requiring a large peak power.

Because of the high frequencies involved in such sonar detection, bats have a hearing system that is specialized to cover this high-frequency range and to provide extended frequency resolution near the signal frequency, perhaps to aid in the neural equivalent of "dechirping" the signal and detecting Doppler frequency shifts. The auditory sensitivity curve of bats is similar in shape to that of humans (threshold about $20 \mu \mathrm{Pa}$ ), but shifted up in frequency by a factor of about 20 to give high sensitivity in the range $10-100 \mathrm{kHz}$ [19.1]. The threshold curve generally shows a particular sensitivity maximum near the frequency of the echo-location call, as might be expected. Bats also generally have forward-facing ears that are large in comparison with their body size. The functions of these in terms of increased directionality and larger receiving area are clear [19.30].

Sound production in bats is generally similar to that in other animals, but with the system dimensions tailored to the higher frequencies involved, and often with the sound emitted through the nose rather than the mouth. The complicated geometry of the nasal tract, which has several spaced enlargements along its length, appears to act as an acoustic filter which suppresses the fundamental and third harmonic of the emitted sound while reinforcing the second harmonic, at least in some species [19.44]. The physics of this arrangement is very similar to that of matching stubs applied to high-frequency transmission lines.

3500. This leads to some very significant differences in the auditory anatomy and behavior between aquatic and air-dwelling animals.

The variety of aquatic animal species is comparable to that of land-dwelling animals, and they can be divided into three main categories for the purposes of the present chapter. First come the crustaceans, such as shrimp, lobsters and crabs, which are analogous to insects, then the wide variety of fish with backbones, and finally the air-breathing aquatic mammals such as the 
cetaceans (whales, dolphins and porpoises, which are the subject of Chap. 19), though animals such as turtles also come into this category. There are, of course, many other categories of marine animal life, but they are not generally significant from an acoustic point of view. A good review has been given by Hawkins and Myrberg [19.45].

The crustaceans, like most insects, generally produce sound by rubbing a toothed leg-file against one of the plates covering their body, thus producing a pulse of rhythmic clicks. Some animals, such as shrimp, may produce a single loud click or pop by snapping their claws in much the same way that a human can do with fingers. Because this activity takes place under water, which has density $\rho$ about 800 times greater and sound velocity $c$ about 4.4 times greater than that of air, and thus a specific acoustic impedance $\rho c$ about 3500 times greater than air, the displacement amplitude of the vibration does not need to be large, and the vibrating structure is generally small and stiff. The peak radiated acoustic power in the "click" can, however, be very large - as much as several watts in the Pistol shrimp Alpheidae probably from a mechanism involving acoustic cavitation. The click lasts for only a very short time, however, so that the total radiated energy is small.

Fish species that lack an air-filled "swim-bladder", the main purpose of which is to provide buoyancy, must produce sound in a similar manner. For fish that do have a swim-bladder, however, the sound production process is much more like that of the cicada, with some sort of plate or membrane over the bladder, or even the bladder wall itself, being set into oscillation by muscular effort and its displacement being aided by the compressibility of the air contained in the bladder. The surrounding tissue and water provide a very substantial mass loading which also contributes to determining the primary resonance frequency of the bladder. This is a much more efficient sound production process than that of the crustaceans and leads to much louder sounds, again generally consisting of a train of repetitive pulses at a frequency typically in the range $100-1000 \mathrm{~Hz}$.

When it comes to sound detection, hair cells again come into play in a variety of ways. Because the density of the animal body is very similar to that of the surrounding water, a plane wave of sound tends to simply move the animal and the water in just the same way. The slight difference in density, however, does allow for some differential motion. The animal body is, however, relatively rigid, so that its response to diverging waves in the near field is rather different from that of the liquid. Any such relative motion between the body and the surrounding water can be detected by light structures such as hairs protruding from the body of the animal, and these can form the basis of hair-cell acoustic detectors. Even more than this, since such cells are generally sensitive to hair deflection in just one direction, arrays of hair cells can be structured so as to give information about the direction of origin of the sound. Although some such detector hairs may be mechanically strong and protrude from the body of the animal, others may be very small and delicate and may be protected from damage by being located in trench-like structures, these structures being called lateral-line organs. Their geometry also has an effect on sound localization sensitivity.

There is another type of hair detector that responds to acceleration rather than relative displacement and that therefore takes advantage of the fact that the body of the animal tends to follow the acoustic displacement of the surrounding water. This is the otolith, which consists of a small dense calcareous stone supported by one or more hair cells, as shown in Fig. 19.9. Since the motion of this stone tends to lag behind the motion of the liquid, and thus of its supports, the supporting hairs are deflected in synchrony with the acoustic stimulus, so that it can be detected by the cells to which they are attached. These sensors will normally have evolved so that combined mass of the otolith and stiffness of the hair give a resonance frequency in the range of interest to the animal, and detection sensitivity will be greatest at this frequency.

If the fish possesses an air-filled swim-bladder, then this can also be incorporated in the hearing sys-

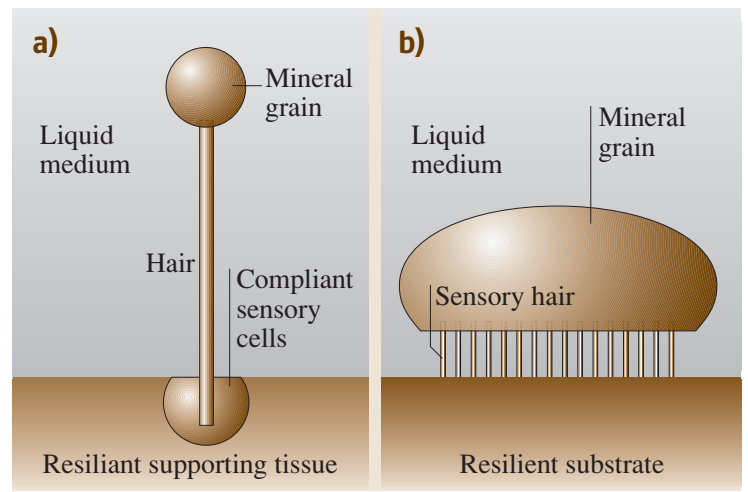

Fig. 19.9a,b Two possible forms for a simple otolith detector: (a) a single-cell detector, (b) a multi-cell detector. As the mineral grain moves sideways relative to the substrate, the sensory hairs are deflected and open ion channels in the supporting transducer cells (from [19.9]) 
tem [19.46]. An air bubble in water is made to move in a sound wave by the differential pressure across it. Because its mass is small and the pressure gradient is the same as that on an equivalent volume of water, the bubble tends to move more than the surrounding liquid but, in order to do so, it must displace a significant amount of this liquid. The final result is that the amplitude of free bubble motion is about three times the amplitude of the motion of the surrounding liquid. Since the body of the fish tends to follow the liquid motion because its density is nearly the same, there is relative motion between the bladder and the rest of the fish, provided it is not rigidly enclosed. This relative motion can be detected by neural cells pressed against the bladder wall and provides an efficient sensor of underwater sound. The radial compressive resonances of the swim bladder can also be important in the detection of sound and, since these are also involved in sound production in these fish, there is a good match between call frequency and the frequency of maximum hearing sensitivity, which typically lies in the range 100 to $1000 \mathrm{~Hz}$. Fish with swim bladders have thus evolved to use these primarily buoyancy-regulating structures for both the production and detection of sound.

\subsection{Generalities}

This brief survey has shown the strong resemblance between the basic sound-producing and auditory mechanisms in a wide variety of animals. Certainly there is a distinction in the matter of sound production between those animals that store air within their bodies and those that do not, and a rather similar distinction in the matter of sound detection, but the similarities are more striking than the differences. In particular, the universal importance of hair-cells in effecting the transduction from acoustic to neural information is most striking.

Also notable in its generality is the scaling of the dominant frequency of produced sound and the

\subsection{Quantitative System Analysis}

Biological systems are almost always anatomically complex, and this is certainly true of vocal and auditory systems, which typically comprise sets of interconnecting tubes, horns, cavities and membranes driven over a wide frequency range by some sort of internal or external excitation. While a qualitative description of the way in which such a system works can be helpful, this must be largely based upon conjectures unless one is able to produce a quantitative model for its behavior. Fortunately a method has been developed for the construction of detailed analytical models for such systems from which their acoustic behavior can be quantitatively predicted, thus allowing detailed comparison with experiment. This section will outline how this can be done, more detail being given in [19.9] and [19.20].

In a simple mechanical system consisting of masses and springs, the quantities of interest are the force $F_{i}$

applied at point $i$ and the resulting velocity of motion $v_{j}$ at point $j$, and we can define the mechanical impedance $Z^{\text {mech }}$ by the equation $Z_{j j}^{\text {mech }}=F_{j} / v_{j}$. Note that the subscripts on $F$ and $v$ are here both the same, so that $Z_{j j}^{\text {mech }}$ is the ratio of these two quantities measured at a single point $j$. It is also possible to define a quantity $Z_{i j}^{\text {mech }}=F_{i} / v_{j}$ which is called the transfer impedance between the two points $i$ and $j$. There is an important theorem called the reciprocity theorem, which shows that $Z_{j i}=Z_{i j}$.

In such a simple mechanical system, as well as the masses and springs, there is normally some sort of loss mechanism, usually associated with internal viscoustype losses in the springs, and the situation of interest involves the application of an oscillating force of magnitude $F$ and angular frequency $\omega$. We denote this force in complex notation as $F \mathrm{e}^{\mathrm{i} \omega t}$ where $\mathrm{i}=\sqrt{-1}$. (Note 
that, in the interest of consistency throughout this Handbook, this notation differs from the standard electrical engineering notation in which $i$ is replaced by j.) For the motion of a simple mass $m$ under the influence of a force $F$ we know that $F=m \mathrm{~d} v / \mathrm{d} t$ or $F=\mathrm{i} \omega m v$, so that the impedance provided by the mass is i $\omega m$. Similarly, for a spring of compliance $C, F=\int v \mathrm{~d} t / C=-\mathrm{i} /(\omega C)$, giving an impedance $-\mathrm{i} /(\omega C)$. For a viscous loss within the spring there is an equivalent resistance $R$ proportional to the speed of motion, so that $F=R v$. Examination of these equations shows that they are closely similar to those for electric circuits if we assume that voltage is the electrical analog of force and current the analog of velocity. The analog of a mass is thus an inductance of magnitude $m$, the analog of a compliant spring is a capacitance $C$, and the analog of a viscous resistance is an electrical resistance $R$. A frictional resistance is much more complicated to model since its magnitude is constant once sliding occurs but changes direction with the direction of motion, so that its whole behavior is nonlinear.

These mechanical elements can then be combined into a simple circuit, two examples being shown in Fig. 19.10. In example (a) it can be seen that the motion of the mass, the top of the spring, and the viscous drag are all the same, so that in the electrical analog the same current must flow through each, implying a simple series circuit as in (b). The total input impedance is therefore the sum of the three component impedances, so that

$$
Z^{\text {mech }}=R+\mathrm{i} \omega m-\frac{\mathrm{i}}{\omega C}=R+\frac{\mathrm{i} m}{\omega}\left(\omega^{2}-\frac{1}{m C}\right),
$$

which has an impedance minimum, and thus maximum motion, at the resonance frequency $\omega^{*}=1 /(m C)^{1 / 2}$. In example (c), the displacement of the top of the spring is the sum of the compressive displacement of the spring and the motion of the suspended mass, and so the analog current is split between the spring on the one hand and the mass on the other, the viscous drag having the same motion as the spring, leading to the parallel resonant circuit shown in (d). There is an impedance maximum, and thus minimum motion of the top of the spring, at the resonance frequency, which is again $\omega^{*}=1 /(m C)^{1 / 2}$. In a simple variant of these models, the spring could be undamped and the mass immersed in a viscous fluid, in which case the resistance $R$ would be in series with the inductance rather than the capacitance. These analogs can be extended to include things such as levers, which appear as electrical transformers with turns ratio equal to the length ratio of the lever arms.

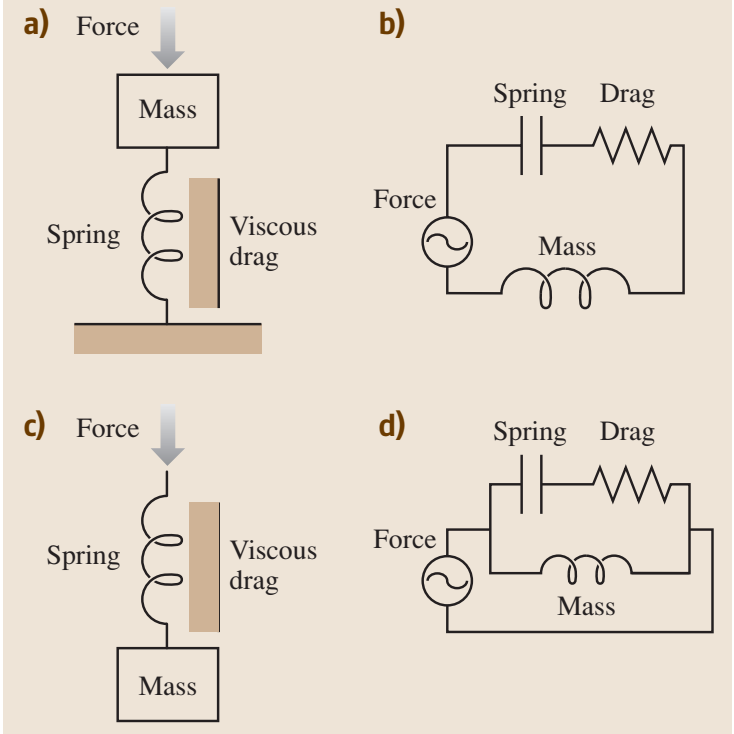

Fig.19.10 (a) A simple resonant assembly with an oscillating force applied to a mass that is resting on a rigidly supported spring, the spring having internal viscous losses. (b) Analog electric network for the system shown in (a). (c) An alternative assembly with the mass being freely suspended from the spring and the oscillating force being applied to the free end of the spring. (d) Analog electric network for the system shown in (c). (Note the potential source of confusion in that an electrical inductance symbol looks like a physical spring but actually represents a mass)

While such combinations of mechanical elements are important in some aspects of animal acoustics, such as the motion of otolith detectors, most of the systems of interest involve the generation and detection of acoustic waves rather than of mechanical vibrations. The important physical variables are then the acoustic pressure $p$ and the acoustic volume flow $U$, both being functions of the frequency $\omega$. By analogy with mechanical systems, the acoustic impedance is now defined as $Z^{\text {acoust }}=p / U$. For a system with a well-defined measurement aperture of area $S$, it is clear that $p=F / S$ and $U=v S$, so that $Z^{\text {acoust }}=Z^{\text {mech }} / S^{2}$, and once again we can define both the impedance at an aperture and the transfer impedance between two apertures. In what follows, the superscript "acoust" will be omitted for simplicity.

Consider first the case of a circular diaphragm under elastic tension and excited by an acoustic pressure $p \mathrm{e}^{\mathrm{i} \omega t}$ on one side. This problem can easily be solved using a simple differential equation [19.9], but here we seek a network analog. The pressure will deflect the dia- 
phragm into a nearly spherical-cap shape, displacing an air volume as it does so. If the small mass of the deflected air is neglected, then deflection of the diaphragm will be opposed by its own mass inertia, by the tension force and by internal losses. The mass contributes an acoustic inertance (or inductance in the network analog) with magnitude about $2 \rho_{\mathrm{S}} d / S$, where $\rho_{\mathrm{s}}$ is the density of the membrane material, $d$ is the membrane thickness, and $S$ is its area, while the tension force $T$ contributes an equivalent capacitance of magnitude about $2 S^{2} / T$. This predicts a resonance frequency $\omega^{*} \approx 0.5\left(T / S \rho_{\mathrm{s}} d\right)^{1 / 2}$, while the rigorously derived frequency for the first mode of the diaphragm replaces the 0.5 with 0.38 . Because biological membranes are not uniform in thickness and are usually loaded by neural transducers, however, it is not appropriate to worry unduly about the exact values of numerical factors. For a membrane there are also higher modes with nodal diameters and nodal circles, but these are of almost negligible importance in biological systems.

The next thing to consider is the behavior of cavities, apertures, and short tubes. The air enclosed in a rigid container of volume $V$ can be compressed by the addition of a further small volume $\mathrm{d} V$ of air and the pressure will then rise by an amount $p_{0} \gamma \mathrm{d} V / V$ where $p_{0}$ is atmospheric pressure and $\gamma=1.4$ is the ratio of specific heats of air at constant pressure and constant volume. The electric analog for this case is a capacitance of value $\gamma p_{0} / V=\rho c^{2} / V$ where $c$ is the speed of sound in air and $\rho$ is the density of air.

In the case of a tube of length $l$ and cross-section $S$, both very much less than the wavelength of the sound, the air within the tube behaves like a mass $\rho l S$ that is set into motion by the pressure difference $p$ across it. The acoustic inertance, or analog inductance, is then $Z=$ $\rho l / S$. For a very short tube, such as a simple aperture, the motion induced in the air just outside the two ends must be considered as well, and this adds an "end-correction" of $0.6-0.8$ times the tube radius [19.47] or about $0.4 S^{1 / 2}$ to each end of the tube, thus increasing its effective length by twice this amount.

These elements can be combined simply by considering the fact that acoustic flow must be continuous and conserved. Thus a Helmholtz resonator, which consists of a rigid enclosure with a simple aperture, is modeled as a capacitor and an inductor in series, with some resistance added due to the viscosity of the air moving through the aperture and to a less extent to radiation resistance. The analog circuit is thus just as in Fig. 19.10b and leads to a simple resonance.

If any of the components in the system under analysis is not negligibly small compared with the wavelength of

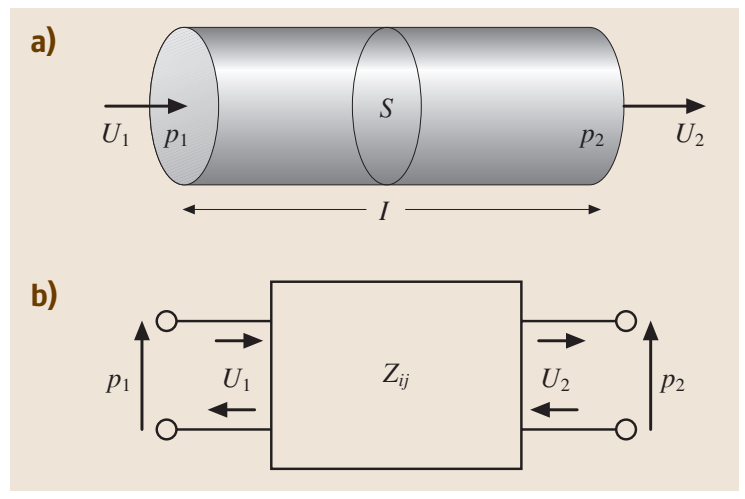

Fig. 19.11 (a) A simple uniform tube showing the acoustic pressures $p_{i}$ and acoustic volume flows $U_{i}$. (b) The electrical analog impedance element $Z_{i j}$. Note that in both cases the current flow has been assumed to be antisymmetric rather than symmetric

the sound involved, then it is necessary to use a more complex analysis. For the case of a uniform tube of length $l$ and cross section $S$, the pressure and flow at one end can be denoted by $p_{1}$ and $U_{1}$ respectively and those at the other end by $p_{2}$ and $U_{2}$. The analog impedance is then a four-terminal element, as shown in Fig. 19.11. The equations describing the behavior of this element are

$$
\begin{aligned}
& p_{1}=Z_{11} U_{1}-Z_{12} U_{2}, \\
& p_{2}=Z_{21} U_{1}-Z_{22} U_{2} .
\end{aligned}
$$

Note that the acoustic flow is taken to be inwards at the first aperture and outwards at the second, and this asymmetry gives rise to the minus signs in (19.8). Some texts take both currents to be inflowing, which removes the minus signs. For a simple uniform tube, symmetry demands that $Z_{21}=Z_{12}$ and that $Z_{22}=Z_{11}$. The first of these relations, known as reciprocity, is universal and does not depend upon symmetry, while the second is not true for asymmetric tubes or horns.

For a uniform tube of length $l$ and cross-section $S$, consideration of wave propagation between the two ends shows that

$$
\begin{aligned}
& Z_{11}=Z_{22}=-\mathrm{i} Z_{0} \cot k l, \\
& Z_{21}=Z_{12}=-\mathrm{i} Z_{0} \csc k l,
\end{aligned}
$$

where $Z_{0}=\rho c / S$ is the characteristic impedance of the tube. The propagation number $k$ is given by

$$
k=\frac{\omega}{c}-\mathrm{i} \alpha
$$


where $\alpha$ represents the attenuation caused by viscous and thermal losses to the tube walls, and has the approximate value

$$
\alpha \approx 10^{-5} \frac{\omega^{1 / 2}}{a}
$$

where $a$ is the tube radius. Since auditory systems often involve quite narrow tubes, this term can become very important.

Similar but much more complicated relations apply for horns of various shapes, the simplest being conical, exponential or parabolic in profile [19.9]. As discussed elsewhere [19.47], horns act as acoustic transformers, basically because $Z_{22} \neq Z_{11}$, and can raise the acoustic pressure in the narrow throat by about the ratio of the mouth diameter to the throat diameter. This amplification is limited, however, to frequencies high enough that the horn diameter does not change greatly over an axial distance of about one wavelength.

With the aid of these electric circuit element analogs it is now possible to construct an analog network to represent the whole acoustic system, as shown by the example in Fig. 19.2. The major guiding principle in constructing such a network is the conservation of acoustic flow, or equivalently of electric current. In the case of an auditory system, there may be several openings connecting to the environment, and the acoustic pressures at these openings will generally differ in both phase and amplitude, depending upon the direction from which the sound is coming. Phase differences are generally more important for directional discrimination than are amplitude differences, particularly for small animals, just as in directional microphones, [19.47] and the combination of tubes, cavities and membranes in the system provides additional phase shifts. The sensory input is normally generated by the motion of tympanic membranes on the two sides of the animal, with the combination of external and internal pressure determining the motion of each membrane, as shown for example in Figs. 19.7 and 19.8.

When sound production is considered, it is usual to make the approximation of separating the basic soundgeneration mechanism, such as the air flow through vibrating vocal folds, from the acoustics of the subsequent tubes and cavities, thus leading to a "source/filter model". With this simplification, the source can be assumed to produce an oscillating acoustic flow at a particular frequency $\omega$, generally accompanied by a set of harmonics at frequencies $n \omega$ generated by nonlinearity in the vibration of the flow valve. The sound production at each frequency is then determined by the final acoustic flow $U(n \omega)$ through an aperture into the surroundings. The square of this flow, multiplied the acoustic radiation resistance of the aperture, which depends upon its size, [19.47] then determines the radiated acoustic power at that frequency. In a much more complex model, the pressure generated by the acoustic input flow can be recognized to act back upon the acoustic source itself, thus modifying its behavior. Typically this results in a small shift in the source frequency to align it more closely with a neighboring strong resonance of the filter. Such interactions are well known in other systems such as musical wind instruments and probably assist in coupling the oscillations of the syrinx to the resonance of the vocal filter in "pure tone" birdsong.

Finally, it is worthwhile to emphasize that biological acoustic systems do not consist of straight tubes of uniform diameter with rigid walls, unloaded circular membranes, or other idealized elements. While it is possible in principle to include such refinements in a computational model, this will generally make that model so complex to analyze that it reveals little about the actual operation of the system. It is usually better, therefore, to construct the simplest possible model that includes all the relevant acoustic elements and to examine its predictions and the effect that changing the dimensions of some of those elements has on the overall behavior. In this way the behavior of the real biological system can be largely understood.

\section{Remark}

The number of books and papers published in this field is very large, so that the reference list below is a rather eclectic selection. Generally descriptive and behavioral references have not been included, and the list concentrates on those that apply physical and mathematical analysis to bioacoustic systems.

\section{References}

19.1 W.C. Stebbins: The Acoustic Sense of Animals (Harvard Univ. Press, Cambridge 1983)

19.2 J.W. Bradbury, S.L. Vehrenkamp: Principles of Animal Communication (Sinauer, Sunderland 1998)
19.3 R.-G. Busnel (Ed.): Acoustic Behavior of Animals (Elsevier, New York 1963)

19.4 A.N. Popper, R.R. Fay (Eds.): Comparative Studies of Hearing in Vertebrates (Springer, Berlin, New York 1980) 
19.5 B. Lewis (Ed.): Bioacoustics: A Comparative Approach (Academic, London 1983)

19.6 D.B. Webster, R.R. Fay, A.N. Popper (Eds.): The Evolutionary Biology of Hearing (Springer, Berlin, New York 1992)

19.7 R.R. Fay, A.N. Popper (Eds.): Comparative Hearing: Mammals (Springer, Berlin, New York 1994)

19.8 R.R. Hoy, A.N. Popper, R.R. Fay (Eds.): Comparative Hearing: Insects (Springer, Berlin, New York 1998)

19.9 N.H. Fletcher: Acoustic Systems in Biology (0xford Univ. Press, New York 1992)

19.10 D.A.W. Thompson: On Growth and Form (abridged), 2nd edn., ed. by J.T. Bonner (Cambridge Univ. Press, Cambridge 1961)

19.11 G.B. West, J.H. Brown: Life's universal scaling laws, Phys. Today 57(9), 36-42 (2004)

19.12 N.H. Fletcher: A simple frequency-scaling rule for animal communication, J. Acoust. Soc. Am. 115, 2334-2338 (2004)

19.13 M. Garstang, D. Larom, R.Raspet, M. Lindeque: Atmospheric controls on elephant communication, J. Exper. Biol. 198, 939-951 (1995)

19.14 K. McComb, D. Reby, L. Baker, C. Moss, S. Sayialel: Long-distance communication of acoustic cues to social identity in African elephants, Anim. Behav. 65, 317-329 (2003)

19.15 J.H. Brackenbury: Power capabilities of the avian sound-producing system, J. Exp. Biol. 78, 163-166 (1979)

19.16 A.W. Ewing: Arthropod Bioacoustics: Neurobiology and Behavior (Cornell Univ. Press, Ithaca 1989)

19.17 A. Michelsen, H. Nocke: Biophysical aspects of sound communication in insects, Adv. Insect Physiol. 10, 247-296 (1974)

19.18 K. Kalmring, N. Elsner (Eds.): Acoustic and Vibrational Communication in Insects (Parey, Berlin, Hamburg 1985)

19.19 N.H. Fletcher: Acoustical response of hair receptors in insects, J. Comp. Physiol. 127, 185-189 (1978)

19.20 N.H. Fletcher, S. Thwaites: Physical models for the analysis of acoustical systems in biology, Quart. Rev. Biophys. 12(1), 25-65 (1979)

19.21 H.C. Bennet-Clark: Resonators in insect sound production: how insects produce loud pure-tone songs, J. Exp. Biol. 202, 3347-3357 (1999)

19.22 B. Dumortier: Morphology of sound emission apparatus in arthropoda. In: Acoustic Behaviour of Animals, ed. by R.-G. Busnel (Elsevier, Amsterdam 1963) pp. 277-345

19.23 B. Dumortier: The physical characteristics of sound emission in arthropoda. In: Acoustic Behaviour of Animals, ed. by R.-G. Busnel (Elsevier, Amsterdam 1963) pp. 346-373

19.24 A.G. Daws, H.C. Bennet-Clark, N.H. Fletcher: The mechanism of tuning of the mole cricket singing burrow, Bioacoust. 7, 81-117 (1996)
19.25 K. Ishizaka, J.L. Flanagan: Synthesis of voiced sounds from a two-mass model of the vocal cords, Bell Syst. Tech. J. 51(6), 1233-1268 (1972)

19.26 N.H. Fletcher: Bird song - a quantitative acoustic model, J. Theor. Biol. 135, 455-481 (1988)

19.27 H.L.F. Helmholtz: On the Sensations of Tone as a Physiological Basis for the Theory of Music (Dover, New York 1954), trans. A.J. Ellis

19.28 G. von Békésy: Experiments in Hearing (McGrawHill, New York 1960)

19.29 E.R. Lewis, E.L. Leverenz, W.S. Bialek: The Vertebrate Inner Ear (CRC, Boca Raton 1985)

19.30 N.H. Fletcher, S. Thwaites: Obliquely truncated simple horns: idealized models for vertebrate pinnae, Acustica 65, 194-204 (1988)

19.31 P.M. Narins, A.S. Feng, W. Lin, H.-U. Schnitzler, A. Densinger, R.A. Suthers, C.-H. Xu: Old world frog and bird vocalizations contain prominent ultrasonic harmonics, J. Acoust. Soc. Am. 115, 910-913 (2004)

19.32 C.H. Greenewalt: Bird Song: Acoustics and Physiology (Smithsonian Inst. Press, Washington 1968)

19.33 D.E. Kroodsma, E.H. Miller (Eds.): Acoustic Communication in Birds, Vol.1-2 (Academic, New York 1982)

19.34 N.H. Fletcher, A. Tarnopolsky: Acoustics of the avian vocal tract, J. Acoust. Soc. Am. 105, 35-49 (1999)

19.35 F. Goller, 0.N. Larsen: A new mechanism of sound generation in songbirds, Proc. Nat. Acad. Sci. U.S.A. 94, 14787-14791 (1997)

19.36 G.B. Mindlin, T.J. Gardner, F. Goller, R.A. Suthers: Experimental support for a model of birdsong production, Phys. Rev. E 68, 041908 (2003)

19.37 D.K. Patterson, I.M. Pepperberg: A comparative study of human and parrot phonation: Acoustic and articulatory correlates of vowels, J. Acoust. Soc. Am. 96, 634-648 (1994)

19.38 G.J.L. Beckers, B.S. Nelson, R.A. Suthers: Vocaltract filtering by lingual articulation in a parrot, Current Biol. 14, 1592-1597 (2004)

19.39 0.N. Larsen, F. Goller: Role of syringeal vibrations in bird vocalizations, Proc. R. Soc. London B 266, 1609-1615 (1999)

19.40 G.J.L. Beckers, R.A. Suthers, C. ten Cate: Pure-tone birdsong by resonant filtering of harmonic overtones, Proc. Nat. Acad. Sci. U.S.A. 100, 7372-7376 (2003)

19.41 N.H. Fletcher, T. Riede, G.A. Beckers, R.A. Suthers: Vocal tract filtering and the "coo" of doves, J. Acoust. Soc. Am. 116, 3750-3756 (2004)

19.42 C. Pytte, M.S. Ficken, A. Moiseff: Ultrasonic singing by the blue-throated hummingbird: A comparison between production and perception, J. Comp. Physiol. A 190, 665-673 (2004)

19.43 G. Sales, D. Pye: Ultrasonic Communication in Animals (Chapman Hall, London 1974) 
19.44 D.J. Hartley, R.A. Suthers: The acoustics of the vocal tract in the horseshoe bat, Rhinolophus hildebrandti, J. Acoust. Soc. Am. 84, 1201-1213 (1988)

19.45 A.D. Hawkins, A.A. Myrberg: Hearing and sound communication under water. In: Bioacoustics: A Comparative Approach, ed. by B. Lewis (Academic, London 1983) pp. 347-405
19.46 N.A.M. Schellart, A.N. Popper: Functional aspects of the evolution of the auditory system in Actinopterygian fish. In: The Evolutionary Biology of Hearing, ed. by D.B. Webster, R.R. Fay, A.N. Popper (Springer, Berlin, New York 1992) pp. 295-322

19.47 T.D. Rossing, N.H. Fletcher: Principles of Vibration and Sound (Springer, New York 2004), Chapts. 8-10 